\title{
ОЦЕНКА КАЧЕСТВА ЖИЗНИ ПРИ ПРИМЕНЕНИИ РАЗЛИЧНЫХ СХЕМ ТЕРАПИИ КЛИМАКТЕРИЧЕСКОГО И ПОСТОВАРЭКТОМИЧЕСКОГО СИНДРОМОВ
}

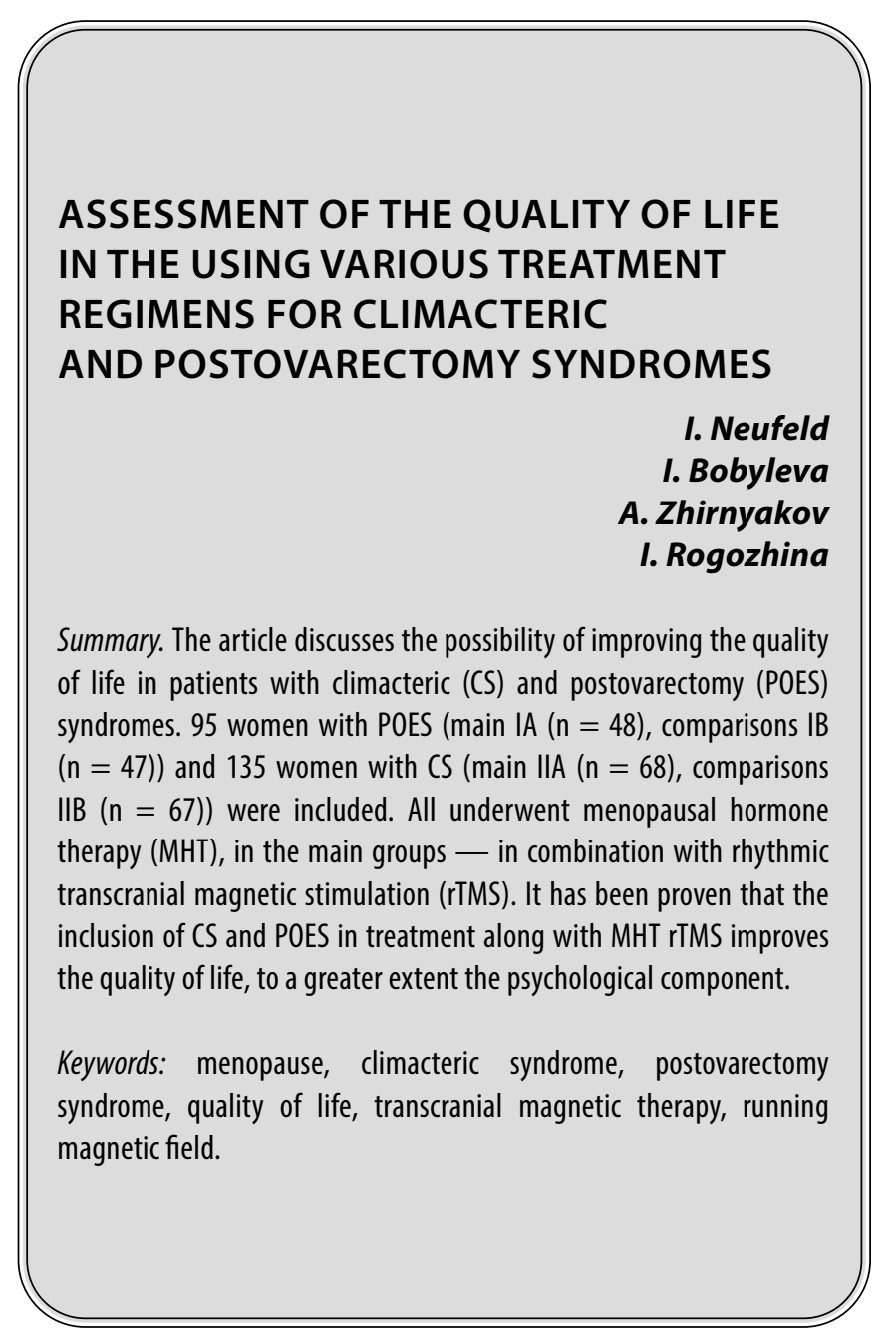

$\mathbf{0}$ тмечающаяся в развитых странах мира инверсия возрастной «пирамиды» обусловлена общим повышением продолжительности жизни и приводит к росту популяции людей старшего возраста, следовательно, закономерно увеличивает длительность пребывания женщины в периоде климактерия.

Климактерий, не являясь собственно заболеванием, обуславливает возникновение и доминирование симптомов, свидетельствующих о дефиците половых стероидов и нередко включает в себя приливы, ночные поты, эмоциональную лабильность, тревожность, нарушение сна, урогенитальные расстройства, снижение либидо и сексуальности, повышение риска развития
Нейфельд Ирина Вольдемаровна

К.м.н., дочент, ФГБОУ ВО «Саратовский государственный медицинский университет им. В.И. Разумовского» МЗ РФ iwn1812@yandex.ru

Бобылева Ирина Владимировна Врач-акушер-гинеколог, ООО «ММК «СОВА», Саратов bobyleva_i@mail.ru

Жирняков Антон Ильич

Дочент, ФГБОУ ВО «Тамбовский государственный университет имени Г.Р. Державина» anton.zhirnyakov@mail.ru

Рогожина Ирина Евгеньевна

Д.м.н., nрофессор, ФГБОУ ВО «Саратовский государственный медицинский университет им. В.И. Разумовского» МЗ РФ kafed-ra@yandex.ru

Аннотация. В статье рассматривается возможность повышения качества жизни у пациенток при климактерическом (KC) и постоварэктомическом (ПОЭС) синдромах. Включено 95 женщин с ПОЭС (основная IA ( $n=48)$, сравнения IB $(n=47))$ и 135 - с КС (основная IIA $(n=68)$, сравнения IIB $(n=67))$. Всем проводилась менопаузальная гормональная терапия (МГТ), в основных группах — В сочетании с ритмической транскраниальной магнитной стимуляцией (рТМС). Доказано, что включение в лечение КС и ПОЭС наряду с МГТ рТМС повышает качество жизни, в большей степени психологического компонента.

Ключевые слова: менопауза, климактерический синдром, постоварэктомический синдром, качество жизни, транскраниальная магнитотерапия, бегущее магнитное поле.

сердечно-сосудистых, обменно-эндокринных заболеваний и остеопороза [1].

При хирургической менопаузе, в частности вследствие тотальной оварэктомии, на фоне остро возникшего эстрогенодефицита, как правило, возникает так называемый постоварэктомический синдром (ПОЭС), клинически схожий с климактерическим синдромом (КС) [1].

Безусловно, наиболее эффективным патогенетическим методом лечения климактерических расстройств при КС и ПОЭС, а также профилактики обменных нарушений является менопаузальная гормональная терапия (МГТ) [1,2]. При этом, как подчеркнуто в российских 
и международных клинических рекомендациях, гормональная терапия должна являться лишь частью общей стратегии сохранения и поддержания здоровья женщин, включающей рекомендации, касающиеся образа жизни, диеты, адекватной физической активности, отказа от курения и злоупотребления алкоголем [2].

Более того в рекомендациях российских и международных организаций по коррекции менопаузальных расстройств в числе главных принципов значится оптимизация качества жизни и обеспечение мероприятий, направленных на долгосрочное благополучие женщин [2-4]. К сожалению, нередко акушеры-гинекологи к числу «общих» относят вопросы, касающиеся качества жизни. При этом вопрос об изменении качества жизни, к примеру, согласно общепринятой лечебной тактике следует задать пациентке, пришедшей на консультацию через два-три месяца от начала МГТ, чтобы оценить эффективность и переносимость назначенного ранее лечения. Однако до сих пор вопросам снижения качества жизни женщин уделяется недостаточно внимания [1-4], несмотря на то, что снижение этого показателя вследствие разнообразных менопаузальных симптомов фактически является главной причиной обращения женщин за медицинской помощью.

Учитывая то, что около 75\% женщин отмечают положительное влияние МГТ на имеющиеся у них симптомы [4], а также то, что использование МГТ ограничено из-за имеющихся абсолютных и относительных противопоказаний к ее назначению, актуальным является не только разработка безопасных подходов к коррекции климактерических расстройств, но и поиск таких способов терапии, которые действовали бы на несколько звеньев патогенеза КС, ПОЭС и обеспечивали как лечебный эффект, так и положительный эффект последействия.

В гинекологической практике имеется опыт применения методик немедикаментозной коррекции климактерических расстройств, зарекомендовавших себя эффективными в коррекции нарушений оси гипотоламус-гипофиз-яичники и способных оказывать модулирующее воздействие на вегетативную нервную систему и на психоэмоциональный статус $[1,5]$.

Несмотря на имеющиеся теоретические предпосылки, ритмическая транскраниальная магнитная стимуляция (рТМС) низкоинтенсивным бегущим полем до настоящего времени не нашла должного применения в лечении пациенток с КС [5]. Более того в доступных нам источниках мы не нашли указания на применение ее в лечебных схемах у пациенток с ПОЭС.

Исходя из вышесказанного, очевидна актуальность анализа эффективности и безопасности применения
рТМС низкоинтенсивным бегущим полем в сочетании с препаратами МГТ при терапии климактерических расстройств у пациенток с КС и ПОЭС.

Цель настоящего исследования - провести сравнительный анализ оценки эффективности и влияния на качество жизни пациенток с КС и ПОЭС монорежима препаратами МГТ и комбинации препаратов МГТ с рТМС низкоинтенсивным бегущим магнитным полем.

\section{Материал и методы}

В настоящей работе нами было обследовано и пролечено 230 пациенток с климактерическими расстройствами, из которых у 95 человек они отмечались при хирургической постменопаузе, у 135 человек - при естественной постменопаузе.

В зависимости от проводимого лечения пациентки случайным образом были распределены в группы основную и сравнения. Таким образом нами были сформированы в случаях хирургической менопаузы - основная группа IA $(n=48)$, и группа сравнения IB $(n=47)$, в случаях естественной менопаузы - основная группа IIA $(n=68)$ и группа сравнения IIB $(n=67)$.

Пациентки обеих групп в целях коррекции климактерических расстройств получали МГТ: при хирургической постменопаузе - эстрадиол гемигидрат ежедневно в лекарственной форме трансдермального геля; при естественной - эстрадиол гемигидрат трансдермально и микронизированный прогестерон 100 мг внутрь в непрерывном режиме.

Пациенткам основной группы добавлялся курс рTMC бегущим импульсным магнитным полем, состоящим из 10 процедур, проводимых через день. Транскраниальная магнитотерапия бегущим импульсным магнитным полем проводилась с помощью физиотерапевтического аппарата «АМО-АТОС-Э» с применением приставки «Оголовье» (Рег. Уд. Минздрава РФ № ФСР 2009 / 04781 от 06.05.2009, производство ООО «ТРИMA», г. Саратов). Частота модуляции для первых двух процедур устанавливалась 1 Гц в течение 5-ти минут, с последующим постепенным увеличением экспозиции на 1 минуту, до 10 минут и частоты модуляции до 10 Гц, курсом 10 дней.

Оценка тяжести климактерических расстройств проводилась по индексу Купермана (1959) в модификации Е.В. Уваровой [1], рассчитывался модифицированный менопаузальный индекс (ММИ).

Оценка качества жизни больных проводилась при помощи опросника «SF-36 Health Status Survey» (SF-36). 


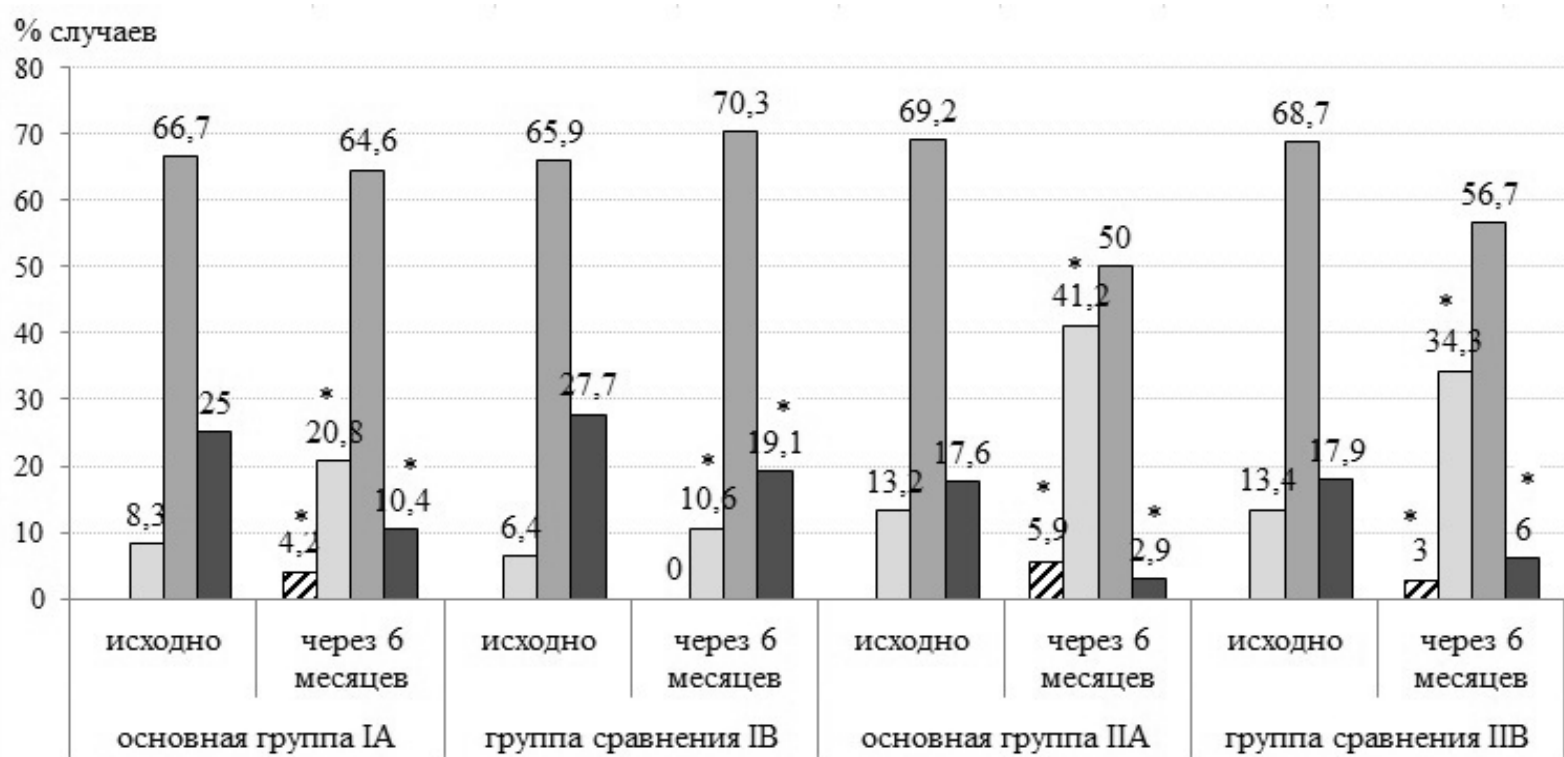

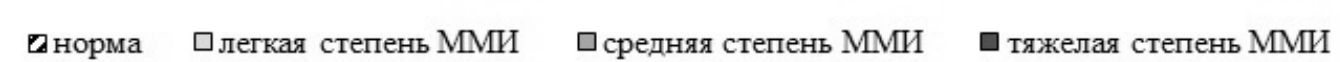

Примечание: * статистически значимые различия по отношению к исходным данным до лечения.

Рис. 1. Сравнительная характеристика динамики количества случаев (в \%) среди пациенток с ПОЭС и КС в ходе лечения

Показатели каждой шкалы варьируют между 0 и 100, где 100 соответствует полному здоровью.

Статистический анализ проводился с использованием программного пакета Statistica (версия 7.0.). Проверка гипотезы на нормальность исходных данных выполнена с помощью критерия Колмогорова-Смирнова, при этом распределение признаков было нормальным. Результаты представляли в виде средних значений (M) и стандартного отклонения (SD). При оценке качественных показателей вычисляли абсолютные и относительные частоты наблюдений (абс.,\%). Различия между двумя средними значениями параметров оценивали по t-критерию Стьюдента, качественными - по критерию Х2 Фишера (статистически значимыми считали различия при $p<0,05)$.

\section{Результаты и их обсужление}

На момент обследования средний возраст пациен-

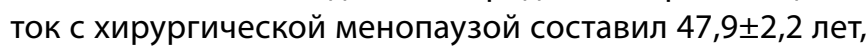
с естественной $-53,6 \pm 1,7$ года $(p=0,042)$; в среднем хирургическая менопауза наступила в 47,1土1,1 лет, естественная - в 51,8 $\pm 1,5$ год $(p=0,012)$. Несмотря на вышеуказанные различия, женщины, включенные с менопаузальными расстройствами в исследование, были сравнимы по длительности эстрогенодефицитного состояния, которое составило $2,8 \pm 0,6$ года $(p=1,04)$.
Все пациентки исходно предъявляли жалобы на нейровегетативные, психоэмоциональные, обменно-эндокринные нарушения, при этом значения ММИ у пациенток рассматриваемых групп значимо различались между собой: при ПОЭС - 57,8土1,1 баллов, при $\mathrm{KC}-49,7 \pm 0,9$ баллов ( $<<0,001)$.

Заслуживает внимание факт того, что проводимая в группах терапия способствовала уменьшению проявлений климактерических расстройств у всех женщин, однако у пациенток в случаях лечения препаратами МГТ в сочетании с рТМС стволовых структур низкоинтенсивным бегущим магнитным полем динамика параметров ММИ была более значимая как при ПОЭС, так и при КС (рис. 1).

Так, через полгода терапии пациенток с ПОЭС увеличение числа случаев с легкой степенью ММИ в основной группе ІА относительно исходных данных произошло в 2,5 раза, в то время как в группе сравнения IB в 1,7 раз; динамика числа случаев пациенток со средней степенью ММИ была не значимой как в основной группе IA, так и в группе сравнения IB; уменьшение количества пациенток с тяжелой степенью ММИ в основной группе ІА отмечено в 2,4 раза, в группе сравнения IB в 1,4 раз (рис. 1).

Лечение менопаузальных расстройств через 6 месяцев у пациенток с КС привело к возрастанию количества случаев с легкой степенью ММИ в основной группе IIA относительно исходных данных в 3,1 раза, в то вре- 
Таблица 1. Динамика общих параметров качества жизни по опроснику SF-36 у пациенток рассматриваемых групп

\begin{tabular}{|c|c|c|c|c|c|c|}
\hline \multirow{3}{*}{$\begin{array}{l}\text { Изучаемые } \\
\text { параметры }\end{array}$} & $\begin{array}{l}\text { основная группа } \\
\text { IA (n=48) }\end{array}$ & $\begin{array}{l}\text { группа сравнения } \\
\text { IB }(n=47)\end{array}$ & $\begin{array}{l}\text { основная группа } \\
\text { IIA (n=68) }\end{array}$ & $\begin{array}{l}\text { группа сравнения } \\
\text { IIB }(\mathbf{n = 6 7 )}\end{array}$ & \multirow{3}{*}{$\mathbf{p}$} & \multirow{3}{*}{$\mathbf{p}^{3-4}$} \\
\hline & 1 & 2 & 3 & 4 & & \\
\hline & M (SD) & M (SD) & $M(S D)$ & M (SD) & & \\
\hline \multicolumn{7}{|c|}{ общий физический компонент здоровья: } \\
\hline исходно & $57,2(2,08)$ & $57,2(3,28)$ & $68,4(2,52)$ & $68,3(3,44)$ & 0,99 & 0,98 \\
\hline через 6 месяцев & $\begin{array}{l}67,9(1,15)^{*} \\
p<0,01\end{array}$ & $\begin{array}{l}64,5(1,13)^{*} \\
p=0,038\end{array}$ & $\begin{array}{l}80,5(1,15)^{*} \\
p<0,01\end{array}$ & $\begin{array}{l}76,5(1,16)^{*} \\
p=0,026\end{array}$ & 0,03 & 0,01 \\
\hline \multicolumn{7}{|c|}{ общий психологический компонент здоровья: } \\
\hline исходно & $53,9(3,66)$ & $53,9(3,86)$ & $64,1(4,62)$ & $64,1(4,12)$ & 0,99 & 0,99 \\
\hline через 6 месяцев & $\begin{array}{l}74,2(3,43)^{*} \\
p<0,01\end{array}$ & $\begin{array}{l}63,9(3,11)^{*} \\
p=0,047\end{array}$ & $\begin{array}{l}86,7(2,44)^{*} \\
p<0,01\end{array}$ & $\begin{array}{l}75,3(2,07)^{*} \\
p=0,017\end{array}$ & 0,03 & $<0,01$ \\
\hline
\end{tabular}

Примечание: * статистически значимые различия по отношению к исходным данным до лечения.

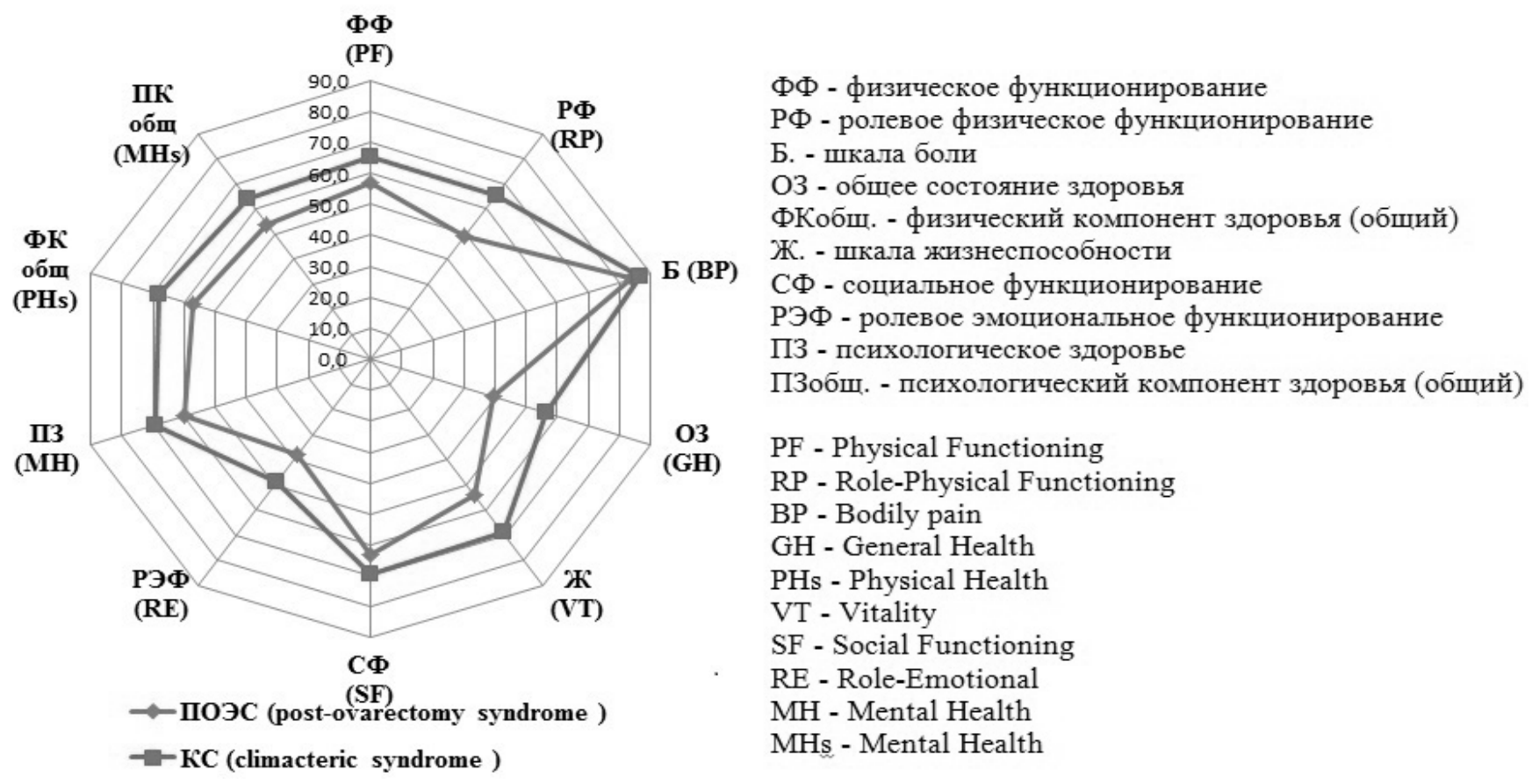

Рис. 2. Сравнительная характеристика исходных параметров качества жизни у пациенток с ПОЭС и КС по шкале опросника SF-36

мя как в группе сравнения IIB в 2,6 раз; динамика числа случаев пациенток со средней степенью ММИ была не значимой как в основной группе IIA, так и в группе сравнения IIB; уменьшение количества пациенток с тяжелой степенью ММИ в основной группе IIA отмечено в 6 раз, в группе сравнения IIB в 3 раза относительно исходных данных (рис. 1).

Обращает на себя внимание и факт того, что состояние полного клинического улучшения через 6 месяцев терапии достигнуто в 4,2\% случаев в основной группе IA, в 5,9\% случаев в основной группе IA (рис. 1). В то вре- мя как у пациенток на фоне монорежима препаратами МГТ нормализация клинической картины через 6 месяцев либо не достигнута ни в одном случае (как в группе сравнения IB у женщин с ПОЭС), либо в меньшем проценте случаев, в частности в $3 \%$ случаев (как в группе сравнения IIB у женщин с KC).

Исходно на фоне имеющихся клинических проявлений эстрогенодефицита у рассматриваемого нами контингента женщин отмечалось снижение показателей качества жизни по всем шкалам опросника SF-36, что наглядно представлено на рисунке 2. 
В динамике лечения пациенток рассматриваемых нами групп изменения по интегративным шкалам опросника оценки качества жизни SF-36 физический и психологический компонент здоровья несколько различались (табл. 1).

В ходе проводимого лечения по шкалам физического и психологического компонентов здоровья общая динамика изменения показателей была положительной у пациенток и с ПОЭС и с КС (табл. 1), однако у пациенток основной группы IA и основной группы ІІА отмечались более высокие значения через 6 месяцев терапии по сравнению с аналогичными параметрами у пациенток группы сравнения IB и группы сравнения IIB.

\section{BыBOА}

Климактерический синдром и в большей степени постоварэктомический синдром у женщин в ранней постменопаузе сопровождаются снижением качества жизни, как по шкалам физического функционирования, так и психологического.

Схема лечения КС и ПОЭС препаратами менопаузальной гормональной терапии с включением курсов ритмической транскраниальной стимуляции стволовых структур низкоинтенсивным бегущим магнитным полем по сравнению с монотерапией препаратами МГТ эффективнее улучшает параметры качества жизни, при этом более значимые изменения достигаются по шкале, составляющей психологический компонент здоровья.

Включение ритмической транскраниальной стимуляции стволовых структур низкоинтенсивным бегущим магнитным полем в практические алгоритмы комплексной терапии менопаузальных расстройств дает возможность повысить эффективность лечения как климактерического, так и постоварэктомического синдромов.

1. Сметник В.П. Медицина климактерия. М.: Литтера, 2009; 848 с.

2. Юренева С.В. Современные подходы к коррекции менопаузальных расстройств. Москва: ГЭОТАР-Медиа, 2017; 80 с.

3. Манухин И.Б. Качество жизни и климактерий. Москва: Литтерра, 2015; 320 с.

4. Gartoulla P, Bell R, Worsley R, Davis S. Menopausal vasomotor symptoms are associated with poor self-assessed work ability. Maturitas 2016; 87: 33-39.

5. Алисултанова Л.С., Бобкова А.С., Кочетков А.В., Орехова Э.М. Нейротропная магнитотерапия в лечении больных с климактерическим синдромом. АГ-Инфо (журнал российской ассоциации акушеров-гинекологов) 2008; (4): 34-37.)

○ Нейфельд Ирина Вольдемаровна ( iwn1812@yandex.ru ), Бобылева Ирина Владимировна ( bobyleva_i@mail.ru ),

Жирняков Антон Ильич ( anton.zhirnyakov@mail.ru ), Рогожина Ирина Евгеньевна ( kafed-ra@yandex.ru ).

Журнал «Современная наука: актуальные проблемы теории и практики» 